\title{
Seismic entangled patterns analyzed via multiresolution decomposition
}

\author{
F. E. A. Leite ${ }^{1}$, R. Montagne ${ }^{2}$, G. Corso ${ }^{3}$, and L. S. Lucena ${ }^{4}$ \\ ${ }^{1}$ Departamento de Ciências Ambientais, Universidade Federal Rural do Semi-Árido, 59625-900, Mossoró, RN, Brazil \\ ${ }^{2}$ Departamento de Física, Universidade Federal Rural de Pernambuco, Rua Dom Manoel de Medeiros, s/n, 52171-900, \\ Recife, PE, Brazil \\ ${ }^{3}$ Departamento de Biofísica e Farmacologia, Centro de Biociências, Universidade Federal do Rio Grande do Norte, Campus \\ Universitário, 59078-972, Natal, RN, Brazil \\ ${ }^{4}$ Departamento de Física Teórica e Experimental, International Center for Complex Systems, Universidade Federal do Rio \\ Grande do Norte, Campus Universitário, 59078-970, Natal, RN, Brazil
}

Received: 19 September 2008 - Revised: 30 January 2009 - Accepted: 22 February 2009 - Published: 26 March 2009

\begin{abstract}
This article explores a method for distinguishing entangled coherent structures embedded in geophysical images. The original image is decomposed in a series of $j$-scaleimages using multiresolution decomposition. To improve the image processing analysis each $\mathrm{j}$-image is divided in 1 -spacial regions generating set of $(\mathrm{j}, 1)$-regions. At each $(\mathrm{j}, \mathrm{l})$-region we apply a continuous wavelet transform to evaluate $E_{v}$, the spectrum of energy. $E_{v}$ has two maxima in the original data. Otherwise, at each scale $E_{v}$ hast typically one peak. The localization of the peaks changes according to the (j,1)-region. The intensity of the peaks is linked with the presence of coherent structures, or patterns, at the respective $(\mathrm{j}, 1)$-region. The method is successfully applied to distinguish, in scale and region, the ground roll noise from the relevant geologic information in the signal.
\end{abstract}

\section{Introduction}

Seismic image filtering and processing is a very prolific area in geophysics (Yilmaz, 2003). Every year, new methods and new approaches in old techniques come into play (Droujinine, 2006; Leite et al., 2008; Liu, 1999). Wavelet analysis (Welford and Zhang, 2004; Corso et al., 2003; Chakraborty and Okaya, 1995), Karhunen-Loève transform (Montagne and Vasconcelos, 2006a,b; Lu, 2002), Empirical Mode Decomposition (Huang et al., 1999), Neural Networks (Tselentis et al., 2007) are just a few techniques used to clean and improve visualization of seismic data. In this paper we fo-

Correspondence to: R. Montagne (montagne@df.ufrpe.br) cus on one of this major image processing methodology: the wavelet analysis (Kumar and Foufoula-Georgiou, 1997; Torrence and Compo, 1998).

We use wavelet analysis for exploring seismic images composed by two entangled patterns: the ground roll and the actual geologic information. In land based seismic data the ground roll shows a peculiar characteristic: it is the main source of noise that contaminates the actual geologic information, but it is at the same time a coherent structure. The ground roll is a surface wave (Rayleigh dispersive wave) with low frequency and low phase and group velocities. In geophysical image-processing, the ground roll is an undesirable information that need to be cleaned (Yilmaz, 2003). Our method allows a good visualization (in scale) of the two patterns immersed in the image. In this way, the method is useful to separate information from these two patterns (or coherent structures) despite the fact that the patterns have not a clear contour and they are spread all over the image.

We develop a method to distinguish mixed structures immersed in a image. The method is based in the multiresolution analysis and the energy spectrum, at each $\mathrm{j}$-scale, obtained out of it. We divide, for improving the resolution, each j-image in 1-slices generating thus, a set of (j, 1)-regions. Then, we decompose each $(\mathrm{j}, 1)$-region in $v$-voices using a continuous wavelet transformation. The normalized square of the components of these wavelet coefficients is called the energy spectrum. We identify in the energy spectrum along each j-image and regions the presence of an eventual coherent structure. In this way, the coherent structures, mixed in the original image, are splitted in scales and voices according to the regions. Although we are mainly concern on seismic images we emphasize that our method is applicable to other

Published by Copernicus Publications on behalf of the European Geosciences Union and the American Geophysical Union. 
problems where one seeks to detect (and eventually remove) mixed coherent structures embedded in a complex pattern (Bernacchia and Naveau, 2008; Piñuela et al., 2007).

We work out our method on the seismic image shown in Fig. 1. This seismic section corresponds to record 25 of Yilmaz's book (Yilmaz, 2003), available at Center for Wave Phenomena and Colorado School of Mines $(2007)^{1}$. The ground roll appears in this seismic image as a fan-like structure with downward oblique straight lines. On the other hand, the geologic information is mainly carried in the almost horizontal lines (actually flat hyperbolas) of the image. The paper is organized as follows. In the next section we present in some detail the wavelet background of the article, fix the mathematical notation and expose the methodology in detail. Then, we apply our method to a specific situation: seismic data contaminated with ground roll noise. Finally, we present our main conclusions and point future research work.

\section{Mathematical background and methodology}

\subsection{Wavelet analysis and multiresolution}

Much of what has been published on wavelets is of great mathematical complexity (Daubechies, 1992; Mallat, 1998) and thus it can not be easily applied by geophysicists. Here, we present, in a simple form, the minimal required mathematical concepts in order to understand the main ideas. A wavelet is defined as a function $\psi(t) \in L^{2}(\mathbb{R})$ (square integrable functions) with a zero mean, localized in both time and frequency. By dilating and translating this wavelet $\psi(t)$, we produce a family of wavelets:

$\psi_{u, s}(t)=\frac{1}{\sqrt{s}} \psi\left(\frac{t-u}{s}\right)$,

where $u, s \in \mathbb{R}$. Here $u$ is a location parameter and $s$ is the dilation parameter or scale. The Continuous Wavelet Transform of a function $f(t), C W T_{f}(u, s)$, is defined as the inner product of the family of wavelets $\psi_{u, s}(t)$ with $f(t)$. This is given by

$\operatorname{CWT}_{f}(u, s)=\left\langle f, \psi_{u, s}\right\rangle=\int_{-\infty}^{+\infty} f(t) \psi_{u, s}^{*} d t$.

where $\psi^{*}$ denotes the conjugate of $\psi_{u, s}$. In order to implement the wavelet transform on sampled signals we need to discretize the parameters $u$ and $s$. The most common choice is $s=2^{j}$ and $u=n 2^{j}$ with $\mathrm{j}$-, n-integers. For a discrete data array, $f[t]$, the Discrete Wavelet Transform $\operatorname{DWT}_{f}(j, n)$ is done by:

$\operatorname{DWT}_{f}(j, n)=\int_{-\infty}^{+\infty} f[t] \frac{1}{\sqrt{2^{j}}} \psi\left(\frac{t-n 2^{j}}{2^{j}}\right) d t$,

\footnotetext{
$1_{\text {availale at: http://www.cwp.mines.edu/software.html }}$
}

where $n=1,2, \ldots$ is the index of data array. So, the function $f(t)$ can be approximated by

$f(t)=\sum_{j} \sum_{n} \operatorname{DWT}_{f}(j, n) \psi_{j, n}(t)$.

The discrete wavelet transform measures the contribution to $f[t]$ of scale $2^{j}$ at location $n 2^{j}$. These conditions are fairly broad and cover a wide range of situations. This features allows the study of a given signal at different scales. Actually, this kind of analysis, known as MULTIRESOLUTION ANALYSIS (or Multiscale Analysis), study and represents at different resolutions (or scales) the signal being analized. In fact, the multiresolution analysis gives in an efficient way an algorithm for going from one scale to another. In order to add more resolution Grossmann et al. (1989) suggested decompose the signal choosing the parameters $s=a^{j}$, with $a=2^{1 / v}$, where $v$ indicates the number of voices ( $v$ voices by scale) per octave (interval $\left[2^{j}, 2^{j+1}\right]$ ) and $u=n u_{0} 2^{j / v}$ with $u_{0}=1$ and $n$ integer. Using this decomposition we get $\psi$

$\psi_{j, n}^{v}(t)=2^{-j / 2 v} \psi\left(2^{-j / v} t-n\right)$

where $j$ and $n$ are integer. Such decomposition using a multivoice frame enables us to cover the range of scales in smaller steps, giving a more continuous picture. For example, with $v=4$ we obtain discrete scales at $\left(s=1,2^{1 / 4}, 2^{1 / 2}, 2^{3 / 4}, 2,2^{5 / 4}, 2^{3 / 2}, 2^{7 / 4}, 4, \ldots\right)$. In agreement with the notation above, the coefficients $\operatorname{DWT}_{f}(j, n)$ have dimension $m \times n$, where $n$ is dimension of $f$ and $m=j * v$. We define the wavelet energy spectrum of $f$ as

$E_{v}=\sum_{n}\left[\operatorname{DWT}_{f}(v, n)\right]^{2}$.

This idea is developed to characterize properties of seismic data.

\subsection{Methodology}

The seismic image is formed by an array of $\left(N_{x}, N_{y}\right)$, where $N_{x}$ is the number of elements in the horizontal (the number of traces in geophysics) and $N_{y}$ the elements in vertical (the number of samples per trace); usually $N_{y} \gg N_{x}$. We summarize our image processing methodology in three parts: (i) Multiresolution decomposition of the original image in a set of j-images, (ii) Slicing of each $\mathrm{j}$-images in a set of l-regions (or slices), (iii) continuous wavelet analysis at each slice to generate the energy spectrum at the $(\mathrm{j}, 1)$-slice. Next we detail the methodology:

1. Multiresolution analysis. The original image is projected into its several scales using a discrete wavelet transform. As a result the multiresolution analysis generates a set of $\mathrm{N}_{\mathrm{j}}$-scale-images. The number $N_{j}$ is related to the size of the signal $N_{y}$ such that $N_{j}$ is the smallest integer that satisfies $N_{y}<2^{N_{j}}$. A detail analysis of decomposition of data in different scales is found in Mallat (1998). The decomposition process of the data shown in Fig. 1 is visualized, for some scales, in Fig. 2. 


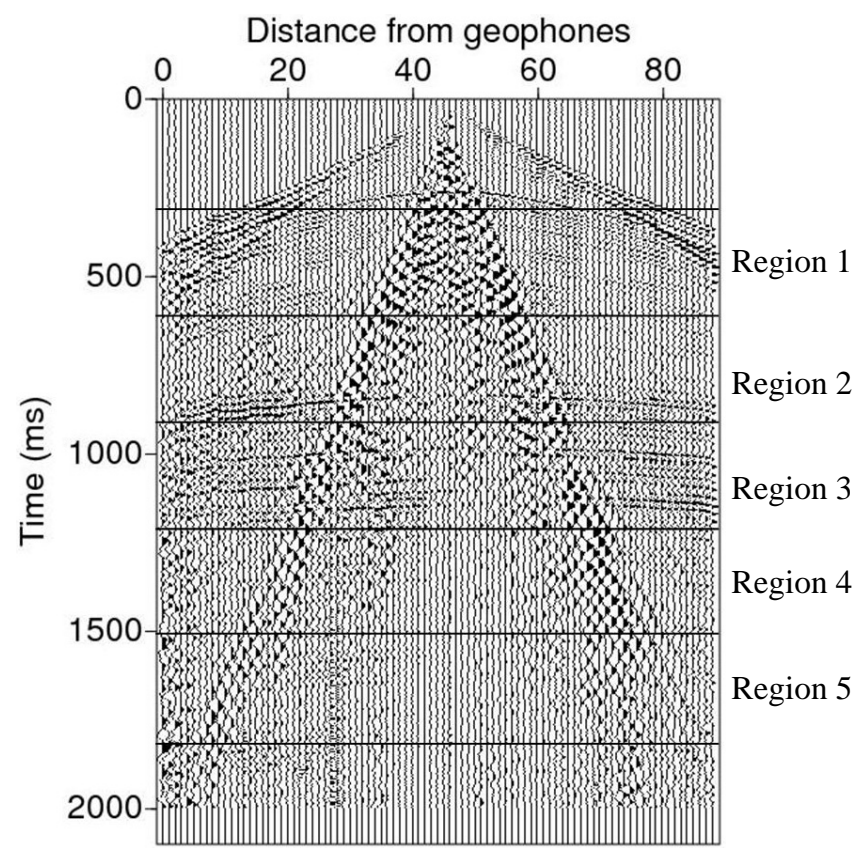

Fig. 1. Original data displaying the ground roll noise as the fan-like structure at the center of the figure. This seismic image corresponds to record 25 in Yilmaz's book. The lines shown on the picture delimit the regions used in the method described in the text.

2. Slicing of the image. Each $\mathrm{j}$-image is sliced in 1-sections for increasing the power of the method, before constructing the energy spectrum. In fact, the analyzed coherent structures are not equally distributed over the full image. As the j-image is sliced the energy spectrum corresponding to the underlying coherent structures at each specific region is emphasized. The number $l$ of slices is empirically determined according to $N_{y}$ and the nature of the data.

3. Energy spectrum. For each $(\mathrm{j}, 1)$-region the signal is decomposed using a continuous wavelet transformation. The energy spectrum is the normalized square of the components of the wavelet transformation. The coherent structures underlying the image are visualized at this step of the method. Typically a coherent structure shows a bump-like signature in the energy spectrum, that means, at each scale the information of the structure is concentrated in a defined set of voices. To summarize, the energy spectrum splits the coherent structures into scales and voices.

\section{Results and discussion}

We apply our methodology for the seismic section shown in Fig. 1. The seismic data has naturally two entangled coher- ent structures: the ground roll and the hyperbolic-like geologic layers. Following the enumerated steps of the previous section, we detail the technical information concerning the application of the method:

1. Multiresolution analysis. We use Daubechies discrete transformation in order to obtain the image at each scale. The size of the data is $N_{y}=2100, N_{x}=89$ which generate $j=11$ scales in the vertical axis.

2. Slicing of the image. We choose to slice Fig. 1, after several tests, in $l=5$ sections. Indeed, larger $l$ does not add new information to the analysis, conversely, smaller $l$, is not enough to explore the localized characteristics of the coherent structures.

3. Energy spectrum. We use the Gaussian wavelet (a continuous wavelet basis) to construct the energy spectrum, with 8 voices. We have also tried the Morlet basis, but the results were not satisfactory.

Figure 2 shows a sequence of four scale-images generated by multiresolution analysis using the discrete wavelet transform. Figure $2 \mathrm{a}-\mathrm{d}$ correspond to scales $j=3,4,5,6$ respectively. We identify in this figure the hyperbolic lines at a and $\mathrm{b}$, on the other hand, the ground roll structure is visible at $\mathrm{d}$. Let us take a closer look at Fig. 2c, it consists of a mix of hyperbolic lines and ground roll patterns. We remark that the mixed image correspond to scale $j=5$, in the next paragraphs we return to this point.

Figure 3 shows a sequence of six energy spectra, the horizontal axis corresponds to the voice $m$ and the vertical to the associated energy $E_{v}$. Each plot shows five curves corresponding to the five slices of the $\mathrm{j}$-images. The slices are taken in a similar way as indicated in Fig. 1. Typically, the peaks in the energy spectrum representation correspond to the coherent patterns of the image. In Fig. 3a we show the spectra of the 5 -slices of the original data. Figure $3 \mathrm{~b}-\mathrm{f}$ correspond to scales $j=3,4,5,6,7$, respectively. A closer look at Fig. 3a reveals that the maxima of the curves are around voices $15-20$ or 25-40 (low versus high voices) depending on the region which produces it. The two peaks shaped curves are due to the presence of an entangled arrangement of patterns: the ground roll (high voices) and the geologic layers (low voices). In fact, at Fig. 3a, regions 2 and 3, that have a well defined geological layers show the peak at low voices. Otherwise, regions 4 and 5, that have strong ground roll presence show the peak at high voices. The bimodal curves correspond to regions 2 and 3, where entangled patterns are visible.

To explore the contents of Fig. 3 we use the following notation. We denote the position of a peak at scale $j$ as $p^{j}$. We also use the index $h$ for peaks corresponding to the hyperbolic lines and index $g$ for ground roll peaks.

Let us take a closer look at the sequence of Fig. 3. We start with low scales (high frequencies). For scale 3 (Fig. 3b) the 


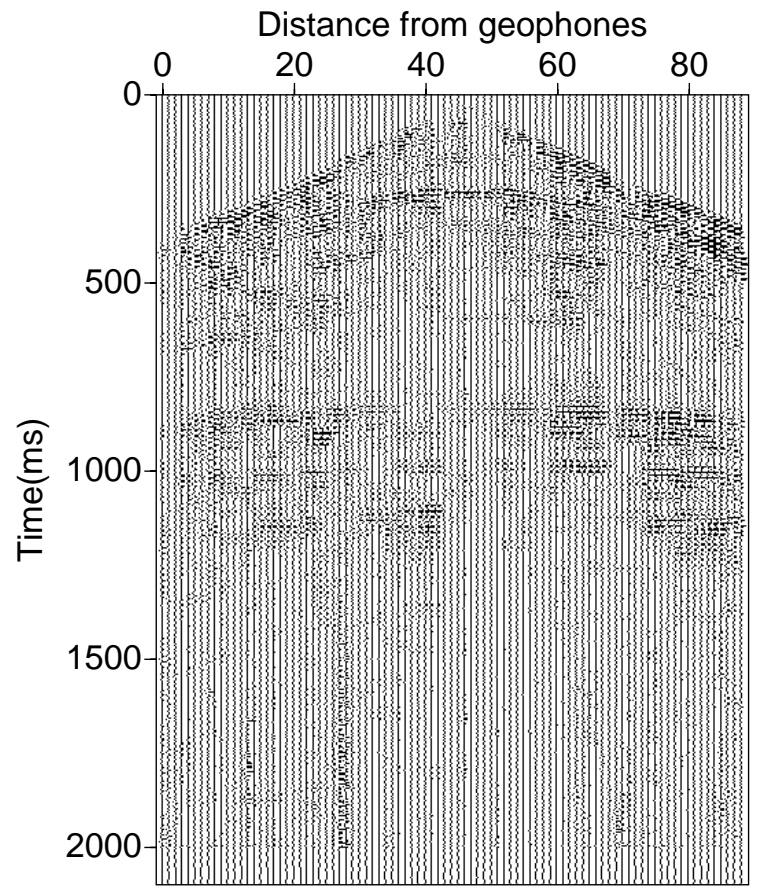

(a)

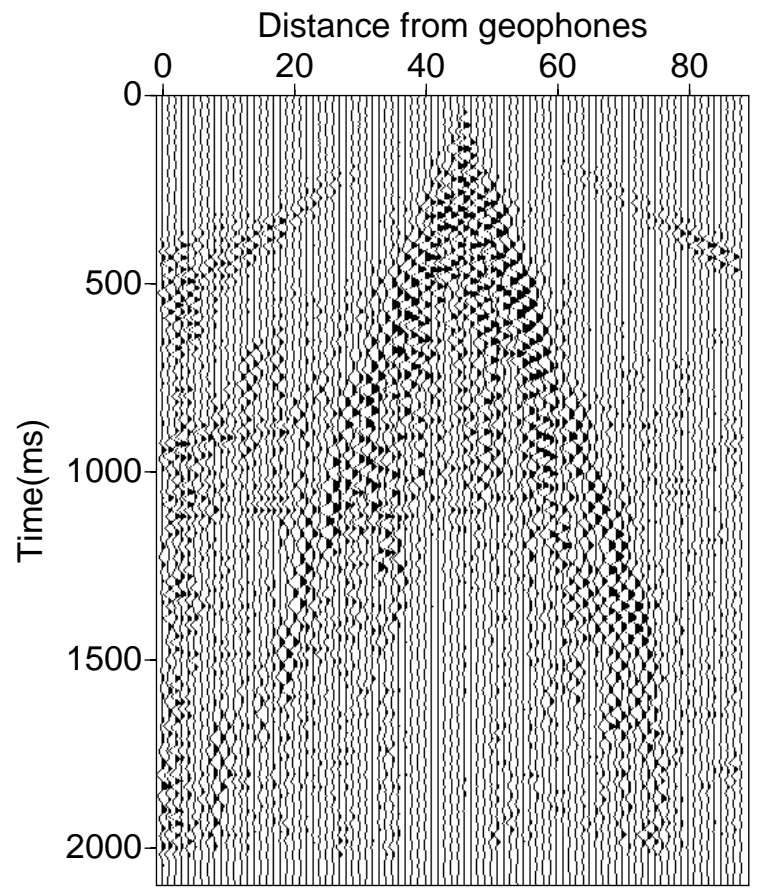

(c)

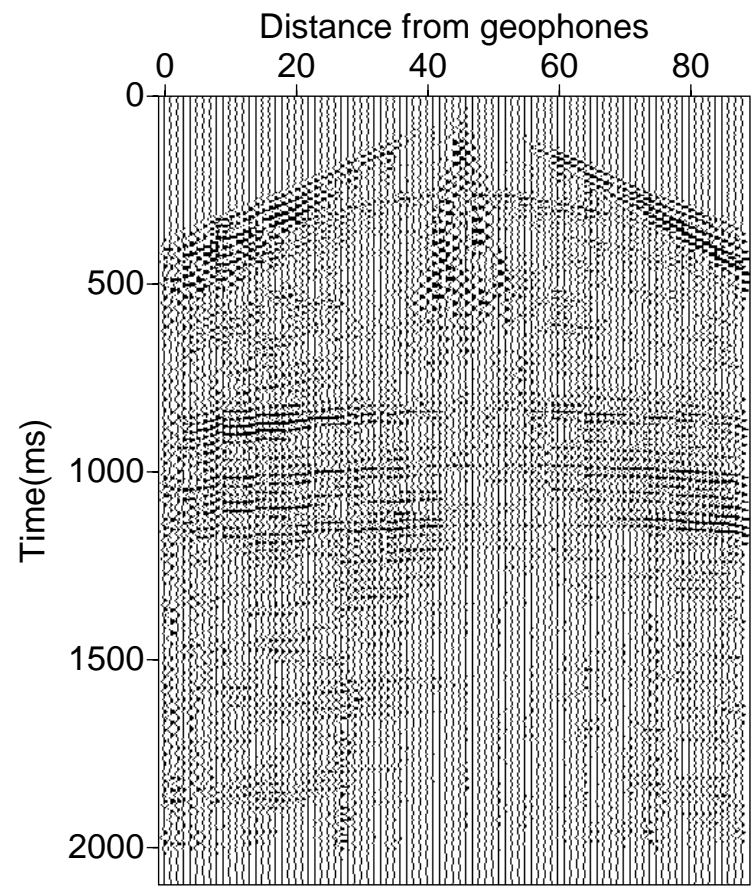

(b)

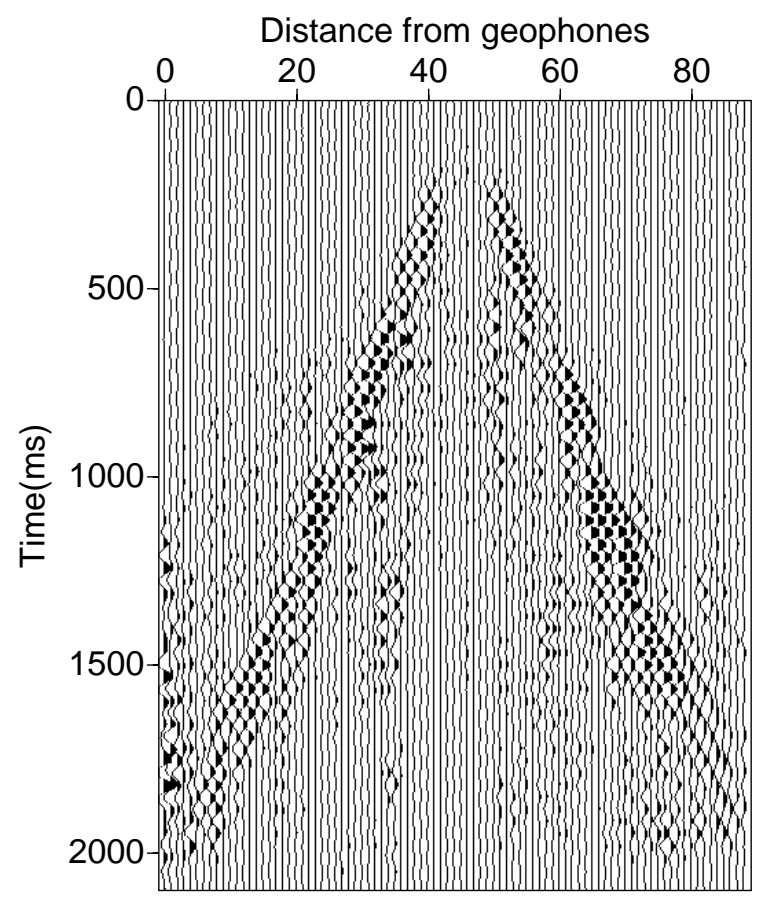

(d)

Fig. 2. Seismic images after multiresolution transformation for scales: (a) $j=3$, (b) $j=4$, (c) $j=5$, and (d) $j=6$. Image (a) and (b) show mainly the hyperbolic lines and image (d) the ground roll. Image (c) shows an entangled arrangement of the two patterns.

peaks corresponding to hyperbolic lines are at $p_{h}^{3}=6$. As can be seen in this figure, all the peaks are at the same position for the 5 regions. The hyperbolic pattern peaks are also visu- alized at $p_{h}^{4}=13$ and $p_{h}^{5}=28$ in Fig. $3 \mathrm{c}$ and d, respectively. We remark that Fig. 3d shows a entanglement of coherent patterns. Indeed, at this scale not only the hyperbolic lines struc- 

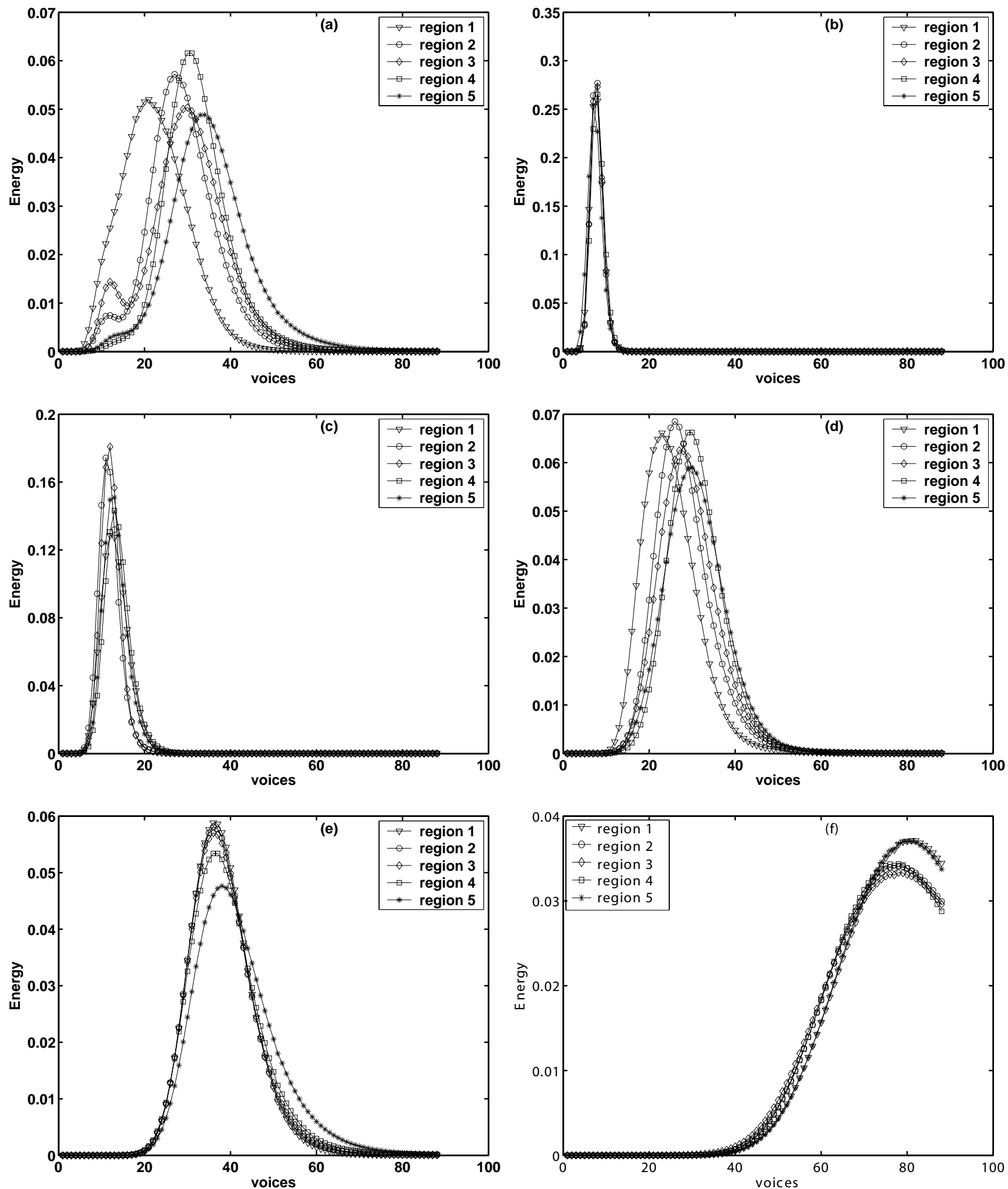

Fig. 3. Energy spectra for several j-images. The different curves in each graphic are to energy spectrum of each region. (a) the original data, (b) $j=3$, (c) $j=4$, (d) $j=5$, (e) $j=6$ and (f) $j=7$. The peaks in the energy spectra are due to the coherent patterns of the images. 
ture is visible, but also the ground roll pattern. The ground roll structure is visualized at high scales (low frequencies). The ground roll peaks are shown in Fig.3d, e and $\mathrm{f}$ at positions $p_{h}^{5}=20, p_{h}^{6}=40$ and $p_{h}^{7}=80$. The reader may well ask why the positions of the peaks due to the ground roll increase by a factor of two as the scale is increased. According to decomposition analysis, the frequency is scaled by a factor of 2 , while going from scale $j$ to $j+1$ that means, the position of a peak at scale $j, p^{j}$, follows the equation: $p^{j+1}=2 p^{j}$.

Remember that, Fig.3d corresponds to a special case, at this scale the peaks of the five regions are not all at the same position. In fact, depending on the region, the ground roll or the hyperbolic lines pattern is the dominant pattern. This fact is reflected in the position of the energy mode peaks. For region 1 the ground roll is more visible and for regions 4 and 5 the peaks are at the same position. At regions 2 and 3 the two patterns are very mixed and the peak is situated at an intermediate position between the previous ones. In the next section we summarize the information concerning this set of figures and conclude the work.

\section{Final remarks}

In this paper we explored the multiresolution analysis for identifying mixed coherent structures immersed in an image. We applied our methodology to seismic data and successfully splitted in voice and scale the two main coherent patterns: the ground roll and the geologic layers. Our main pourpose was to provide an effective method for separating in scales and voices mixed coherent patterns.

The multiresolution decomposition split an image into several scales, or frequency ranges. However, coherent pattern may be not restricted to a single scale. Specifically, in the case we studied, the ground roll pattern is present at scales $j=5,6$ and 7 and the hyperbolic lines pattern at $j=1,2,3,4$ and 5 . The scale $j=5$ is the most stimulating case as it shares information from the two patterns. The patterns in this scale are splitted according to voices, hence, different patterns correspond to peaks centered at distinct voices in the energy spectrum. We clearly visualized this characteristic analyzing separately the regions of the seismic image. The regions corresponding to strong ground roll presence (slices 4 and 5) are associated with peaks at the same position. In contrast, the regions corresponding to hyperbolic patterns ( 2 and 3, mainly 3 ) are related to peaks at a different position in the energy spectra. The region 3 deserves special attention, this region exhibit the most entangled mixing of the two patterns, consequently, the associated energy spectrum shows a peak in between the two former cases.

In this manuscript we present a method for splitting seismic patterns using wavelet decomposition. We remark that we apply the wavelet transform over the data acquired at each geophone, that means, we transform the data of each trace, an unidimensional signal. In this way we do not capture the full spacial information of the seismic image. Because we do not work directly on a bidimensional data we can not use more sophisticated basis like anisotropic Morlet basis or biorthogonal basis (Neupauer and Powell, 2005; Allaei et al., 2008). The success of the splitting patterns in a one dimensional data encourage us to improve the method to perform the wavelet transform on two dimensional data. A natural basis in two dimensional data is the anisotropic Morlet which was specially designed to deal with heterogeneous geologic images.

As a final remark we point out that our method will prove useful to design intelligent coherent pattern filtering. Let us suppose that we are interested in cleaning the ground roll and preserve the hyperbolic lines. In this case we should keep scales 1,2,3,4 and the medium and low voices of the scale 5 . The scales 6 and 7, as well the high voices of scale 5 should be erased. We are working at present, on a coherent pattern filter that automatically decompose the signal, identify the patterns, and erase the inconvenient patterns.

Acknowledgements. Financial support from the Brazilian agencies CNPq, FAPERN, FACEPE, FINEP and CTPETRO is acknowledged.

Edited by: T. Chang

Reviewed by: three anonymous referees

\section{References}

Allaei, S. V., Sahimi, M., and Tabar, M. R. R.: Propagation of acoustic waves as a probe for distinguishing heterogeneous media with short-range and long-range correlations, J. Stat. Mech.Theory E, 3, 16-+, doi:10.1088/1742-5468/2008/03/P03016, 2008.

Bernacchia, A., Naveau, P., Vrac, M., and Yiou, P.: Detecting spatial patterns with the cumulant function - Part 2: An application to El Niño, Nonlin. Processes Geophys., 15, 169-177, 2008, http://www.nonlin-processes-geophys.net/15/169/2008/.

Chakraborty, A. and Okaya, D.: Frequency-time decomposition of seismic data using wavelet-based methods, Geophysics, 60, 1906-1916, 1995.

Corso, G., Kuhn, P., Lucena, L., and Thomé, Z.: Seismic ground roll time-frequency filtering using the Gaussian wavelet transform, Physica A, 318, 551-561, 2003.

Daubechies, I.: Ten Lectures on Wavelets, in: CBMS-NSF Regional Conference Series in Applied Mathematics, SIAM: Society for Industrial and Applied Mathematics, 61, ISBN-13: 9780-898712-74-2/ISBN-10: 0-89871-274-2, 1992.

Droujinine, A.: Multi-scale geophysical data analysis using the eigenimage discrete wavelet transform, J. Geophys. Eng., 3, 5981, 2006.

Grossmann, A., Kronland-Martinet, R., and Morlet, J.: Reading and understanding continuous wavelets transforms, Springer, New York, NY, USA, 2nd edn. 1991, p. 20, 1989.

Huang, N. E., Shen, Z., and Long, S.: A new view of nonlinear water waves: The Hilbert spectrum, Annu. Rev. Fluid Mech., 31, 417-457, 1999.

Kumar, P. and Foufoula-Georgiou, E.: Wavelet analysis for geophysical applications, Rev. Geophys., 35, 385-412, 1997. 
Leite, F., Montagne, R., Corso, G., Vasconcelos, G., and Lucena, L.: Optimal wavelet filter for suppression of coherent noise with an application to seismic data, Physica A, 387(7), 1439-1445, 2008.

Liu, X.: Ground roll suppression using the Karhunen-Loève transform, Geophysics, 64, 564 pp., 1999.

Lu, W.: Adaptive noise attenation of seismic image using Singular Value Decomposition an texture direction detection, IEEE T. Image Process., 2, p. 465, 2002.

Mallat, S.: A wavelet tour of signal processing, Academic Press, New York, 2nd edn., 637 pp., 1998.

Montagne, R. and Vasconcelos, G.: An optimized filter for seismic data using the Karhunen-Loève transform and a minimumenergy criterium, Physical Review E, 74, 01016213, 2006a.

Montagne, R. and Vasconcelos, G.: Thermodynamic criteria for optimal suppression of coherent noise in seismic data using the Karhunen-Loève transform, Physica A, 371(1), 122-125, $2006 \mathrm{~b}$.

Neupauer, R. M. and Powell, K. L.: A fully-anisotropic Morlet wavelet to identify dominant orientations in a porous mediumstar, Comput. Geosci., 31, 465 pp., 2005.
Piñuela, J., Andina, D., McInnes, K., and Tarquis, A.: Wavelet analysis in a structured clay soil using 2-D images, Nonlin. Processes Geophys., 14, 425-434, 2007, http://www.nonlin-processes-geophys.net/14/425/2007/.

Torrence, C. and Compo, G. P.: A Practical Guide to Wavelet Analysis, B. Am. Meteorol. Soc., 79, 61 pp., 1998.

Tselentis, G., Serpetsidaki, A., Martakis, N., Sokos, E., Paraskevopoulos, P., and Kapotas, S.: Local high-resolution passive seismic tomography and Kohonen neural networks - Application at the Rio-Antirio Strait, central Greece, Geophysics, 72(4), B93-B106, 2007.

Welford, J. K. and Zhang, R.: Ground-roll suppression from deep crustal seismic reflection data using a wavelet-based approach: A case study from western Canada, Geophysics, 69(4), p. 877, 2004.

Yilmaz, O.: Seismic Data Processing, Society of Exploration Geophysicists, Tulsa, 2nd edn., 2027 pp., 2003. 